\title{
Análisis de la influencia del cuidador principal en el estado nutricional del paciente en hemodiálisis
}

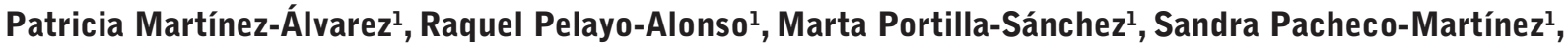 \\ María José Cagigas-Villosladal, José Luis Cobo-Sánchez²,3 \\ ${ }^{1}$ Unidad de Hemodiálisis. Servicio de Nefrología. Hospital Universitario Marqués de Valdecilla. Santander. España \\ ${ }^{2}$ Subdirección de Cuidados. Servicio Cántabro de Salud. Santander. España \\ ${ }^{3}$ Escuela Universitaria de Enfermería Clínica Mompia. Universidad Católica de Ávila. Mompia. España
}

\begin{abstract}
Como citar este artículo: Martínez-Álvarez P, Pelayo-Alonso R, Portilla-Sánchez M, Pacheco-Martínez S, Cagigas-Villoslada MJ, Cobo-Sánchez JL. Análisis de la influencia del cuidador principal en el estado nutricional del paciente en hemodiálisis.

Enferm Nefrol. 2020 0ct-Dic;23(4):381-387
\end{abstract}

\section{Resumen}

Introducción: La población que se encuentra en tratamiento renal sustitutivo con hemodiálisis sufre frecuentemente cierto grado de desnutrición calórico-proteica debido a las restricciones dietéticas y la mala elección de alimentos. Esta desnutrición unida a la inflamación repercute en su calidad de vida e incrementa su mortalidad.

Objetivos: Determinar el estado nutricional de los pacientes en tratamiento con hemodiálisis y su relación con quién elabora su comida. Comprobar la relación entre el grado de cumplimiento de las recomendaciones terapéuticas y su estado nutricional.

Material y Método: estudio transversal y descriptivo en una población de pacientes en hemodiálisis hospitalaria. Se recogieron datos sociodemográficos, antecedentes clínicos, resultados analíticos y antropométricos. El estado nutricional se valoró mediante la escala MIS. Se realizó un análisis descriptivo e inferencial de las variables a estudio.

Resultados: El grado de desnutrición se situó en el $32,4 \%(n=34)$. No se encontró ninguna significación estadística entre el estado nutricional y el cuidador prin-

\section{Correspondencia:}

Patricia Martínez Álvarez

Email: patmaralv@hotmail.com cipal. Pese a que el 76,5\% refirió seguir las recomendaciones dietéticas, tan solo un $44 \%$ tenía unos valores séricos de potasio adecuados y el 34,7\% cumplía con la ganancia de peso interdialítica recomendada.

Conclusiones: La desnutrición sigue siendo un problema frecuente en las unidades de hemodiálisis. Se deben tomar medidas para reducirla, ya sea con acciones educacionales sobre alimentación o incluso valorar el uso de suplementos dietéticos. Enfermería debe seguir reforzando las recomendaciones dietéticas para que ese cumplimiento referido por nuestros pacientes pueda objetivarse y verse reflejado en sus resultados analíticos.

PALABRAS CLAVE: desnutrición; hemodiálisis; dieta; cumplimiento y adherencia al tratamiento; cuidadores.

\section{Analysis of the influence of the primary caregiver in the nutritional status of the patient on hemodialysis}

\section{Abstract}

Introduction: People undergoing renal replacement therapy for hemodialysis frequently suffer a certain degree of caloric-protein malnutrition due to dietary restrictions and poor food choices. Malnutrition together with inflammation affects quality of life and increases mortality. 
Objectives: To determine the nutritional status of patients on hemodialysis and the relationship with who cooks the food. To establish the relationship between the degree of compliance with the therapeutic recommendations and the nutritional status.

Material and Method: Cross-sectional and descriptive study in a population of patients on hospital hemodialysis. Sociodemographic data, clinical history, analytical and anthropometric results were collected. Nutritional status was assessed using the MIS scale. A descriptive and inferential analysis of the variables under study was carried out.

Results: The degree of malnutrition was $32.4 \%(n=34)$. No significant difference was found between nutritional status and the main caregiver. Although $76.5 \%$ reported following the dietary recommendations, only $44 \%$ had adequate serum potassium values and $34.7 \%$ met the recommended inter-dialytic weight gain.

Conclusions: Malnutrition continues to be a frequent problem in hemodialysis units. Measures should be taken to reduce this problem, either with educational actions on nutrition or even evaluating the use of dietary supplements. Nursing must continue to reinforce dietary recommendations so that compliance referred by patients can be objectified and reflected in the analytical results.

KEYWORDS: malnutrition; hemodialysis; diet; treatment adherence and compliance; caregivers.

\section{Introducción}

La población que se encuentra en tratamiento renal sustitutivo con hemodiálisis (HD) sufre frecuentemente de cierto grado de desnutrición calórico-proteica debido a las restricciones dietéticas y la mala elección de alimentos a la hora de elaborar los menús. La bibliografía describe que tanto para los expertos en nutrición como para los pacientes la dieta en HD es una de las dietas más restrictivas y complejas ${ }^{1}$.

La adherencia terapéutica o "cumplimiento", se define con frecuencia como: "los grados en los que las conductas del paciente coinciden con las recomendaciones de los proveedores de atención médica"2.

La menor adherencia terapéutica está relacionada con un mayor riesgo de malnutrición ${ }^{3}$. Esta desnutrición unida a la inflamación repercute en la calidad de vida de
Ios pacientes, aumentando su mortalidad. Por todo ello resulta beneficioso conocer el estado nutricional de los pacientes en tratamiento con HD. Un buen marcador del deterioro nutricional en el paciente hemodializado es la albúmina sérica ${ }^{4}$, por lo que es un valor para tener en consideración en estos pacientes. Las enfermeras son los profesionales que más contacto tiene con los pacientes durante las sesiones de HD. Esta situación supone un momento muy favorable en el que educar al paciente, facilitando la información precisa sobre sus posibles restricciones alimentarias y métodos de cocinado más beneficiosos y recomendados, para lograr una buena alimentación, permitiendo un abordaje individualizado y siempre adaptado a las necesidades de cada paciente ${ }^{5}$. En la actualidad es bastante frecuente encontrarnos con pacientes de edad avanzada que manifiestan una pérdida de apetito y el no disfrutar de la comidas ${ }^{4-6}$. Esta situación, unida a las importantes restricciones alimentarias necesarias provoca que los menús que siguen los pacientes sean poco apetitosos y monótonos, lo que facilita la desnutrición $n^{5,6}$.

La adherencia al tratamiento médico es un proceso complejo influenciado por muchos factores: sociales, individuales, culturales y ambientales6. El apoyo familiar y social son factores facilitadores de la adherencia terapéutica ${ }^{7}$, por eso es necesario involucrar a los cuidadores principales y valorar si un buen apoyo familiar influye en el estado nutricional de nuestros pacientes. El objetivo principal fue determinar el estado nutricional del paciente de los pacientes en tratamiento con HD y su relación con quién elabora su comida. Como objetivo secundario nos planteamos comprobar la relación entre el grado de cumplimiento de las recomendaciones terapéuticas y su estado nutricional.

\section{Material y Método}

Se realizó un estudio descriptivo trasversal en pacientes en tratamiento con HD crónica en la Unidad de HD del Hospital Universitario Marqués de Valdecilla, desde agosto de 2019 a enero de 2020. Se incluyeron a aquellos pacientes que, tras recibir la información pertinente, otorgaron su consentimiento para participar en el estudio. Fueron criterios de exclusión presentar una situación clínica grave 0 un deterioro cognitivo que impidiese un buen nivel de comunicación o tener problemas para comprender el idioma.

Se recogieron datos de la historia clínica electrónica del paciente, incluyendo datos sociodemográficos (edad, 
sexo) y datos clínicos: nefropatía de base según código ERA/EDTA, grado de comorbilidad mediante el índice de Charlson, antecedentes personales de diabetes mellitus (DM), índice de masa corporal (IMC), tiempo en HD; así como parámetros de diálisis, incluyendo dosis de diálisis (mediante valores de $\mathrm{Kt} / \mathrm{V}$ ), ganancia de peso interdialítica (GPI) y parámetros séricos pre HD durante el periodo de estudio (potasio, albúmina, transferrina). Se estableció como indicador de una buena adherencia al régimen terapéutico, cifras de potasio sérico $<5 \mathrm{mEq} / \mathrm{L}$ y una GPI $\leq 2500 \mathrm{ml}$. Se valoró el tipo de convivencia de cada participante, valorando si el paciente vivía solo, con la familia o estaba institucionalizado, así como quién se encargaba de elaborar su comida (si era el propio paciente o no).

El estado nutricional e hídrico del paciente se evaluó con bioimpedancia eléctrica multifrecuencia (determinada con frecuencia mensual pre-HD durante el periodo de estudio); y mediante la escala MIS (Malnutrition Inflamation Score) ${ }^{8}$, escala que valora globalmente la nutrición y la inflamación. Incluye 4 apartados que incluyen 10 parámetros, que se valoran en una escala tipo Likert de 0 a 3 puntos:

a. Antecedentes Personales: cambios en el peso seco en los últimos 6 meses, cambios en la ingesta alimentaria, síntomas gastrointestinales, incapacidad funcional y comorbilidades asociadas.

b. Exploración física: pérdida de tejido adiposo, signos de pérdida de masa muscular.

c. Índice de Masa Corporal (IMC).

d. Parámetros bioquímicos: Albúmina sérica y transferrina.

La suma de todas las puntuaciones determina el estado nutricional. Puntuaciones menores o iguales a 10 establecen que el paciente está bien nutrido.

Al final del periodo de estudio, y referido a los últimos 6 meses, se encuestó al paciente sobre el grado autopercibido de cumplimiento del régimen dietético mediante una pregunta cerrada con las opciones: si, no, en parte (más del $75 \%$ de las ocasiones).

A partir de las variables estudiadas, se elaboró una base de datos tipo Excel. Para el análisis de los datos se utilizó el paquete estadístico PSPP v1.2. Se realizó un análisis descriptivo utilizando medidas de tendencia central para las variables cuanti- tativas y frecuencias y porcentajes para las variables cualitativas. Para la inferencia estadística se utilizaron los test Chi cuadrado, T de Student, ANOVA de un factor y prueba de Kruskal Wallis, considerándose un valor $p<0,05$ como estadísticamente significativo.

El estudio se llevó a cabo cumpliendo la Ley orgánica $3 / 2018$, de 5 de diciembre de protección de datos personales y garantía de los derechos digitales.

\section{Resultados}

Se estudiaron 34 pacientes, siendo hombres el 58,8\% de la muestra. La edad media se situó en $69,5 \pm 15,1$ años (mediana 72 años). El índice de comorbilidad medio fue $4,82 \pm 1,5$ puntos (mediana 5 puntos) y el tiempo medio en tratamiento con HD de $59,82 \pm 77,29$ meses (mediana 25,5 meses; rango 6-358 meses). EI $\mathrm{KT} / \mathrm{V}$ se mostró en valores adecuados con valores medios de $1,68 \pm 0,20$. El resto de las características clínicas de la muestra se especifican en la Tabla 1.
Tabla 1. Características clínicas de la muestra $(n=34)$.

\begin{tabular}{|c|c|c|c|}
\hline & & Frecuencia & Porcentaje \\
\hline Nefropatía base & $\begin{array}{l}\text { Glomerular } \\
\text { Intersticial } \\
\text { Sistémica } \\
\text { Congénita } \\
\text { Otras } \\
\text { Desconocida }\end{array}$ & $\begin{array}{c}9 \\
5 \\
14 \\
3 \\
2 \\
1\end{array}$ & $\begin{array}{c}26,5 \% \\
14,7 \% \\
41,2 \% \\
8,8 \% \\
5,9 \% \\
2,1 \%\end{array}$ \\
\hline Diabetes & $\begin{array}{l}\text { Si } \\
\text { No }\end{array}$ & $\begin{array}{l}10 \\
24\end{array}$ & $\begin{array}{l}29,4 \% \\
70,6 \%\end{array}$ \\
\hline $\begin{array}{l}\text { Adherencia régimen } \\
\text { terapéutico } \\
\text { (según paciente) }\end{array}$ & $\begin{array}{l}\text { Si } \\
\text { No } \\
\text { En parte }\end{array}$ & $\begin{array}{c}26 \\
4 \\
4\end{array}$ & $\begin{array}{l}76,5 \% \\
11,8 \% \\
11,8 \%\end{array}$ \\
\hline Convivencia & $\begin{array}{l}\text { Sólo } \\
\text { Pareja/familia } \\
\text { Institución/empleado }\end{array}$ & $\begin{array}{c}4 \\
28 \\
2\end{array}$ & $\begin{array}{c}11,8 \% \\
82,4 \% \\
5,9 \%\end{array}$ \\
\hline $\begin{array}{l}\text { Encargado de } \\
\text { elaborar la comida }\end{array}$ & $\begin{array}{l}\text { Paciente } \\
\text { Familiar } \\
\text { Institución/empleado }\end{array}$ & $\begin{array}{c}10 \\
21 \\
3\end{array}$ & $\begin{array}{c}29,4 \% \\
61,8 \% \\
8,8 \%\end{array}$ \\
\hline Cocina el paciente & $\begin{array}{l}\text { Si } \\
\text { No }\end{array}$ & $\begin{array}{l}10 \\
24\end{array}$ & $\begin{array}{l}29,4 \% \\
70,6 \%\end{array}$ \\
\hline $\begin{array}{l}\text { Desnutrición } \\
(M I S>10)\end{array}$ & $\begin{array}{l}\text { Si } \\
\text { No }\end{array}$ & $\begin{array}{l}10 \\
24\end{array}$ & $\begin{array}{l}29,4 \% \\
70,6 \%\end{array}$ \\
\hline
\end{tabular}

MIS: Malnutrition Inflamation Score. 
Tabla 2. Cumplimiento terapéutico durante el periodo de estudio.

\begin{tabular}{lccccc}
\hline & MEDIA & DE & MEDIANA & MINIMO & MAXIMO \\
\hline Potasio sérico (mEq/L) & 4,85 & 0,71 & 4,79 & 3,46 & 6,03 \\
\hline analíticas mensuales con no cumplimiento potasio & 44,09 & 39,54 & 33,30 & 0 & 100 \\
GPI (ml) & 2209,26 & 607,84 & 2069,89 & 981,67 & 3537,99 \\
\hline sesiones con no cumplimiento GPI & 34,70 & 30,25 & 25,37 & 0 & 96,15 \\
\hline
\end{tabular}

DE: desviación estándar; GPI: ganancia de peso interdialítica.

En la Tabla 2 se detallan los datos relativos al cumplimiento del régimen terapéutico durante los 6 meses del periodo de estudio. El $76,5 \%$ de los pacientes refirió seguir las recomendaciones dietéticas prescritas, pero tan sólo el $44 \%$ y el $34,7 \%$ de la muestra lograron mantener unos valores adecuados de potasio sérico y de ganancia interdialítica respectivamente en función de los valores límite establecidos como óptimos.

De los pacientes incluidos en el estudio, 24 pacientes presentaron desnutrición, lo que supone el $70,6 \%$ del total de la muestra. No se halló significación estadística entre el estado de desnutrición y el nivel de convivencia del paciente. En la Tabla 3 se detallan las características clínicas de la muestra según el tipo de convivencia del paciente. No se encontró relación entre el cumplimiento del régimen terapéutico y la convivencia del paciente, lo que se detalla en la Tabla 4.
No hubo diferencias estadísticamente significativas entre la puntuación media de la escala MIS y quien se encargaba de elaborar la comida (paciente 7 puntos, familiar 10 puntos, institución/empleado 11 puntos; $p=0,623$ ); y si era el paciente quien cocinaba (cocina el paciente 7,18 puntos vs no cocina paciente 9,91 puntos; $p=0,063$ ). Así mismo, no hubo diferencias estadísticamente significativas entre los clasificados como desnutridos por una puntuación mayor de 10 en la escala MIS y quien se encargaba de elaborar la comida (paciente: desnutridos 8/bien nutridos 2, familiar: desnutridos 17/bien nutridos 4, institución/ empleado: desnutridos 3/bien nutridos $0 ; p=0,925) ; y$ si era el paciente quien cocinaba (cocina el paciente: desnutridos 8/bien nutridos 2 vs no cocina paciente: desnutridos 20/bien nutridos $4 ; p=0,054$ ).

Tampoco hubo diferencias estadísticamente significativas con respecto a la adherencia y quien preparaba la comida:

Tabla 3. Características clínicas del paciente según el tipo de convivencia.

\begin{tabular}{|c|c|c|c|c|c|c|c|c|}
\hline & & \multicolumn{2}{|c|}{$\begin{array}{l}\text { Vive sólo } \\
(n=4)\end{array}$} & \multicolumn{2}{|c|}{$\begin{array}{l}\text { Vive con } \\
\text { pareja/familia } \\
(n=28)\end{array}$} & \multicolumn{2}{|c|}{$\begin{array}{l}\text { Institucionalizado } \\
\qquad(n=2)\end{array}$} & \multirow[t]{2}{*}{$\mathbf{p}$} \\
\hline & & $n$ & $\%$ & $n$ & $\%$ & $n$ & $\%$ & \\
\hline Sexo & $\begin{array}{l}\text { Hombre } \\
\text { Mujer }\end{array}$ & $\begin{array}{l}1 \\
3\end{array}$ & $\begin{array}{l}25 \% \\
75 \%\end{array}$ & $\begin{array}{l}18 \\
10\end{array}$ & $\begin{array}{l}64,3 \% \\
35,7 \%\end{array}$ & $\begin{array}{l}1 \\
1\end{array}$ & $\begin{array}{l}50 \% \\
50 \%\end{array}$ & 0,317 \\
\hline Nefropatía & $\begin{array}{l}\text { Glomerular } \\
\text { Intersticial } \\
\text { Sistémica } \\
\text { Congénita } \\
\text { Otras } \\
\text { Desconocida }\end{array}$ & $\begin{array}{l}1 \\
- \\
3 \\
- \\
- \\
-\end{array}$ & $\begin{array}{c}25 \% \\
- \\
75 \% \\
- \\
- \\
-\end{array}$ & $\begin{array}{c}8 \\
4 \\
10 \\
3 \\
2 \\
1\end{array}$ & $\begin{array}{c}28,6 \% \\
14,3 \% \\
35,7 \% \\
10,7 \% \\
7,1 \% \\
3,6 \%\end{array}$ & $\begin{array}{l}- \\
1 \\
1 \\
- \\
- \\
-\end{array}$ & $\begin{array}{c}- \\
50 \% \\
50 \% \\
- \\
- \\
-\end{array}$ & 0,855 \\
\hline Diabetes & $\begin{array}{l}\text { Si } \\
\text { No }\end{array}$ & $\begin{array}{l}3 \\
1\end{array}$ & $\begin{array}{l}75 \% \\
25 \%\end{array}$ & $\begin{array}{c}6 \\
22\end{array}$ & $\begin{array}{l}21,4 \% \\
78,6 \%\end{array}$ & $\begin{array}{l}1 \\
1\end{array}$ & $\begin{array}{l}50 \% \\
50 \%\end{array}$ & 0,072 \\
\hline $\begin{array}{l}\text { Adherencia al régimen } \\
\text { terapéutico } \\
\text { (según el paciente) }\end{array}$ & $\begin{array}{l}\text { Si } \\
\text { No } \\
\text { En parte }\end{array}$ & $\begin{array}{l}2 \\
2 \\
-\end{array}$ & $\begin{array}{l}50 \% \\
50 \% \\
-\end{array}$ & $\begin{array}{c}23 \\
2 \\
3\end{array}$ & $\begin{array}{c}82,1 \% \\
7,1 \% \\
10,7 \%\end{array}$ & $\begin{array}{l}1 \\
- \\
1\end{array}$ & $\begin{array}{c}50 \% \\
- \\
50 \%\end{array}$ & 0,053 \\
\hline $\begin{array}{l}\text { Desnutrición } \\
(\text { MIS > 10) }\end{array}$ & $\begin{array}{l}\text { Si } \\
\text { No }\end{array}$ & $\begin{array}{l}4 \\
-\end{array}$ & $\begin{array}{c}100 \% \\
-\end{array}$ & $\begin{array}{l}18 \\
10\end{array}$ & $\begin{array}{l}64.3 \% \\
35,7 \%\end{array}$ & $\begin{array}{l}2 \\
-\end{array}$ & $\begin{array}{c}100 \\
-\end{array}$ & 0,458 \\
\hline
\end{tabular}

MIS: Malnutrition Inflamation Score (puntuación de la escala). 
Tabla 4. Características clínicas y cumplimiento del régimen terapéutico en función de la convivencia del paciente.

\begin{tabular}{|c|c|c|c|c|c|c|c|}
\hline & \multicolumn{2}{|c|}{$\begin{array}{c}\text { Vive solo } \\
(n=4)\end{array}$} & \multicolumn{2}{|c|}{$\begin{array}{c}\text { Vive con } \\
\text { pareja/familia } \\
(n=28)\end{array}$} & \multicolumn{2}{|c|}{$\begin{array}{l}\text { Institucionalizado } \\
\qquad(n=2)\end{array}$} & \multirow[t]{2}{*}{$\mathbf{p}$} \\
\hline & Media & $\mathrm{DE}$ & Media & $\mathrm{DE}$ & Media & $\mathrm{DE}$ & \\
\hline Edad (años) & 67 & 14 & 70 & 16 & 63 & 11 & 0,775 \\
\hline IMC $\left(\mathrm{Kg} / \mathrm{m}^{2}\right)$ & 27,37 & 3 & 24,40 & 4,97 & 21,35 & 4,89 & 0,331 \\
\hline Comorbilidad (puntos) & 4 & 1 & 5 & 2 & 5 & 3 & 0,752 \\
\hline Kt/V (litros) & 1,55 & 0,17 & 1,69 & 0,21 & 1,76 & 0,10 & 0,389 \\
\hline Potasio sérico (mEq/l) & 4,49 & 0,15 & 4,88 & 0,73 & 5,12 & 1,15 & 0,520 \\
\hline No cumplimiento potasio (\%) & 25 & 0,96 & 47 & 41,3 & 41,7 & 58,9 & 0,836 \\
\hline GPI $(\mathrm{ml})$ & $2.186,19$ & 986,48 & $2.169,86$ & 525,09 & $2.806,99$ & $1.033,79$ & 0,369 \\
\hline No cumplimiento GPI (\%) & 38,50 & 38,09 & 32,26 & 28,39 & 61,27 & 49,33 & 0,449 \\
\hline Puntuación escala MIS (puntos) & 8,25 & 5,18 & 9,03 & 3,95 & 10,5 & 4,95 & 0,821 \\
\hline
\end{tabular}

DE: Desviación Estándar; IMC: Índice de Masa Corporal; GPI: Ganancia de Peso Interdialítica; MIS: Malnutrition Inflamation Score.

-Porcentaje medio de sesiones con no cumplimiento GPI y quien se encargaba de elaborar la comida (paciente $26,52 \%$, familiar 32,59\%, institución/ empleado $61,27 \% ; p=0,626)$; y si era el paciente quien cocinaba (cocina el paciente $36,26 \%$ vs no cocina paciente $34,04 \% ; p=0,849)$.

-Porcentaje de analíticas mensuales con no cumplimiento potasio y quien se encargaba de elaborar la comida (paciente $25 \%$, familiar $50 \%$, institución/ empleado $41,7 \% ; p=0,626)$; y si era el paciente quien cocinaba (cocina el paciente $26,6 \%$ vs no cocina paciente $51,36 \% ; p=0,097$ ).

\section{Discusión}

Según nuestros datos, la desnutrición es un problema muy prevalente en los pacientes sometidos a HD, misma situación que refleja la bibliografía existente ${ }^{8-12}$. Una valoración continuada del estado nutricional por parte de Enfermería, permitiría controlar el estado nutricional del paciente y así detectar las alteraciones y prevenir las complicaciones ${ }^{7,13}$. El tiempo que pasa la enfermera con el paciente en la sala de diálisis es un gran momento para la intervención educativa y reforzar las restricciones dietéticas que tan necesarias han demostrado ser para prevenir complicaciones y detectar y analizar comorbilidades que puedan requerir de nuestra ayuda o de formación extra al paciente como la diabetes, alteraciones de la deglución o la absorción de nutrientes. El paciente con diabetes y en tratamiento de HD presenta una mayor probabilidad de malnutrición siendo esta de tipo calórica. Sin embargo, en nuestro caso, la prevalencia de diabetes apenas alcanza el $30 \%$.

La escala Malnutrition Inflamation Score (MIS) nos ha demostrado ser un buen instrumento de valoración del estado nutricional, que facilita el cuidado de los pacientes de HD. El tiempo en diálisis y la comorbilidad son los principales aspectos a la hora de controlar el estado nutricional8. La impresión visual de la complexión corporal de los pacientes estables en tratamiento con HD es engañosa y en un alto porcentaje camuflan alteraciones nutricionales ${ }^{14}$.

Uno de los objetivos de nuestro estudio era valorar la posible relación entre el cuidador principal del paciente y su estado nutricional y grado de cumplimiento de las recomendaciones dietéticas, ya que son dos factores que influyen sobre la morbimortalidad de los pacientes. Sin embargo, no hemos encontrado bibliografía que relacione estos dos aspectos.

En la mayoría de las familias, el cuidado de las personas dependientes es asumido por una única persona, actividad que recae con mayor frecuencia en la mujer. Andreu y cols. ${ }^{10}$ en su estudio identifican que 8 de cada 10 cuidadores son mujeres entre 45 y 65 años. La realidad de las salas de HD nos muestra pacientes cada vez más añosos y dependientes ${ }^{11-14}$. El 82,4\% de nuestra muestra vive con su pareja o con la familia y más del $60 \%$ refieren que cocina un familiar, es decir, 
depende de un cuidador principal. Aunque en nuestro estudio no se ha encontrado relación entre el estado nutricional del paciente y la figura del cuidador, lo que se puede deber al escaso tamaño de la muestra, es necesario incluir la figura del cuidador en la educación al paciente, debiendo tenerlos en cuenta a la hora de valorar, modificar y mejorar los hábitos dietéticos de los pacientes en HD.

Según nuestros datos, la desnutrición es un problema muy frecuente en los pacientes de nuestra muestra, probablemente debido a su edad avanzada y al alto índice de comorbilidad que presentan. Se deben tomar medidas para reducir esta desnutrición ya sea con acciones educacionales sobre alimentación o incluso valorar el uso de suplementos dietéticos.

En nuestra muestra, no se encontraron relaciones estadísticamente significativas entre el estado nutricional de los pacientes en tratamiento con HD y su relación con quién elabora su comida. Sin embargo, sí que existían diferencias clínicamente relevantes a favor de las personas que convivían con su pareja/familia y que éstos elaboraban su comida, con un mejor estado nutricional y cumplimiento terapéutico con respecto a la dieta.

Creemos en la necesidad de involucrar al cuidador principal en la educación nutricional, ya que son los encargados de la alimentación de la mayoría de nuestros pacientes.

Enfermería debe seguir reforzando las recomendaciones dietéticas para que el cumplimiento terapéutico que refieren los pacientes sea un dato objetivo y se refleje en sus resultados analíticos.

Recepción:13-10-20

Aceptación: 4-11-20

Publicación: 30-12-20

\section{Bibliografía}

1. Lambert K, Mullan J, Mansfield K. An integrative review of the methodology and findings regarding dietary adherence in end stage kidney disease. BMC Nephrol. 2017;18(1):318.

2. Vitolins MZ, Rand CS, Rapp SR, RibisI PM, Sevick MA. Measuring adherence to behavioral and medical interventions. Control Clin Trials. 2000;21(5, Suppl 1):S188.

3. González JA, Granados SA, Rebollo A, Villegas C, Vicario V, Mansilla Francisco JJ. Adherencia terapéutica relacionada con el estatus nutricional de una cohorte de pacientes en tratamiento renal sustitutivo con hemodiálisis. Enferm Nefrol. 2016;19(Supl 1):S56.

4. Quero Alfonso AI, Fernández Castillo R, Fernández Gallegos R, Gómez Jiménez FJ. Estudio de la albúmina sérica y del índice de masa corporal como marcadores nutricionales en pacientes en hemodiálisis. Nutr. Hosp. 2015;31(3):1317-22.

5. Bernardo C. Adherencia dietética de los pacientes en hemodiálisis. Enfermería CyL. 2019;11(1):96105.

6. Cupisti A, Kalantar-Zadeh K. Management of Natural and Added Dietary Phosphorus Burden in kidney disease. Semin Nephrol. 2013;33(2):180-90.

7. González Oquendo L, Maroto Santana I, Fernández Ramírez C, Cabello Florín I. Valoración del estado nutricional del paciente con insuficiencia renal crónica en hemodiálisis. Metas Enferm. 2014;17(9):50-5.

8. Elvira Carrascal $S$, Colomer Codinachs $M$, Pérez Oller L, Chirveches Pérez E, Puigoriol Juvanteny E, Pajares Requena $D$ et al. Descripción del estado nutricional de los pacientes de una unidad de diálisis mediante el uso de la escala "Malnutrition Inflamation Score". Enferm Nefrol. 2013;16(1):23-30.

9. Gómez Vilaseca L, Manresa Traguany M, Morales Zambrano J, García Monge E, Robles Gea MJ, Chevarria Montesinos JL. Estado nutricional del paciente en hemodiálisis y factores asociados. Enferm Nefrol. 2017;20(2):120-5.

10. Andreu Periz L, Moreno Arroyo MC. Perfil y realidad social de los cuidadores principales de pacientes dependientes tratados con hemodiálisis. Nursing (Ed Esp). 2009;27(4):54-65. 
11. Gómez J. Valoración del estado nutricional de pacientes mayores de 65 años en tratamiento sustitutivo en una unidad de diálisis. Rev Soc Esp Enferm Nefrol. 2006;9(2):84-90.

12. González L, Maroto I, Fernández C, Cabello I. Valoración del estado nutricional del paciente con insuficiencia renal crónica en hemodiálisis. Metas de Enferm. 2014;17(9):50-5.

13. Pereira MC, Queija L, Blanco A, Rivera I A, Martínez V E, Prada Z. Valoración del estado nutricional y consumo alimentario de los pacientes en terapia renal sustitutiva mediante hemodiálisis. Enferm Nefrol. 2015;18(2):103-11.

14. Manzano JM, Nieto MD, Sánchez MC. Valoración nutricional de enfermería de los pacientes tratados con hemodiálisis en un centro periférico. Rev Soc Esp Enferm Nefrol. 2004;7(1):10-8.

Este artículo se distribuye bajo una Licencia Creative Commons Atribución-NoComercial 4.0 Internacional. https://creativecommons.org/licenses/by-nc/4.0/

\section{Open Access C) (i) ()}

